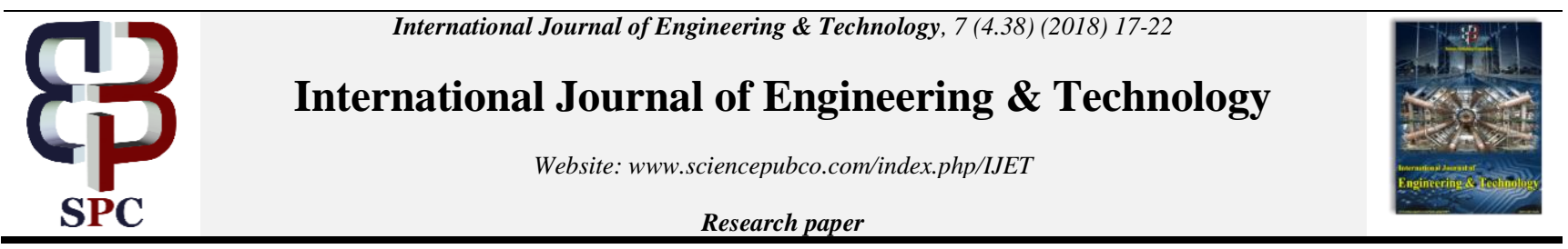

\title{
Design and Analysis for the presence of Bacillus cereus in blood using Plasmons
}

\author{
Ajey S S* P C Srikanth* Dr D N Nagalaxmi** Dr V R Krishnamurthy*** N Chandra Shekar **** \\ *Dept. Of E And C Engineering, Malnad College Of Engineering, \\ Hassan, Karnataka, India \\ **Dept. Of Pathology, Hassan Institute Of Medical Sciences, \\ Hassan, Karnataka, India \\ *** Dept. Of Surgery, Hassan Institute Of Medical Sciences, \\ Hassan, Karnataka, India \\ ****M.Tech (VLSI And Embedded System), CMR Institute Of Technology, BANGALORE, KARNATAKA, INDIA \\ *Corresponding author E-mail: ajey328@gmail.com
}

\begin{abstract}
The Photonic Waveguide biosensors are used for detection and analysis of bio-analyte. These photonic waveguides use Plasmon vibrations technique for detection of the analyte by combining biological components with physicochemical detector. FDTD which stands for finite difference time domain is used for designing a 2- D Surface Plasmonic Resonance based photonic waveguide-based biosensor for detection of the Micro-bacteria named Bacillus cereus in order to find the toxicity in the blood. For modelling and designing the photonic waveguide sensor, Rsoft CAD simulation tool is used. Monitor values, wavelength and Frequency spectrums have been observed and by using these parameters Sensitivity is calculated. A slight variation in the refractive index leads to a substantial change in the frequency which can be seen in the band structure, therefore the designed structure behaves as sensor. Therefore, designed sensor is greatly sensitive to the modifications in RI and hence it discriminates among the dissimilar constituents of the analyte.
\end{abstract}

Keywords - Bacillus Cereus, Biosensor, SPR, FDTD, Sensitivity

\section{Introduction}

Over past two decades there has been a remarkable improvement in the development of biosensors in the field of biotechnology, food safety, drug screening, and security and also in the field of medical diagnostics. The bacillus cereus is being find out by the application of surface plasmon resonance. The SPR optical sensor designed is built on a metal dielectric configuration with a higher sensitivity. Basically, an affinity biosensor involves a transducer which can be a piezoelectric or optical and a bio-recognition element that can interact with the analyte that is selected. Based on the optical means many sensors have been designed in the field of sensing particularly it involves spectroscopy based optical waveguide which involve the guided modes and a SPR Waveguides developed using fluorescence provides high sensitivity since it involves the labeling of molecules they require a multistep detection procedure, this results the sensor to become sensitive to the analyte which is non-targeted. Sensor like grating couplers, SPR and optical interferometer are a label free technology which depends on the measurement of the binding interaction which produces RI change. A Silver $(\mathrm{Ag}) / \mathrm{gold}(\mathrm{Au})$ configuration [1] is suggested in order to attain decent chemical resistance by keeping sensitivity high. The characteristics of the sensor surface are its simulation results when it is used in spectral SPR optical sensors. The results of the simulation insist that finest arrangement for spectral Surface Plasmon Resonance sensors must be $2 \mathrm{~nm}$ for $\mathrm{Au}$ and $48 \mathrm{~nm}$ for $\mathrm{Ag}$ dielectric for accomplishing finest sensitivity with decent chemical resistance.

\section{Theory}

SPR optical sensor are built on a metal dielectric configuration and it [1] affords great sensitivity for numerous operations like sensing of biological and chemical interactions. The most commonly used arrangement in the SPR Sensor is the gold dielectric one because tinny Au layer delivers steady performance and decent chemical resistance. The Fresnel formulas [2][3] is used to study features of spectral Surface Plasmon Resonance sensors which use compound $\mathrm{Au} / \mathrm{Ag}$ films for transducing layer. SPR as an optical sensing method is extensively used for the measurement of biological and chemical quantities. The SPR utilizes finite difference time domain technique. An imagination is made such that region of space doesn't have any isolated charges or flowing currents. The EM waves propagates inside biosensor. The dielectric properties of the salt percentage concentration will change which in turn shifts wavelength. The resonance frequency is gained in graphical format by means of which the exact location of the resonance frequency can be found. The Maxwell's equations [3] are resolved in four regions with suitable boundary conditions put at each stage. The wave propagation is considered only in the z-direction and the variations in the $\mathrm{y}$ direction is ignored.

Considering below equation

$\mathrm{d}^{2} \mathrm{f} / \mathrm{dx}^{2}\left(k^{2} 0 n^{2}-\beta^{2}\right) \emptyset=0$ 
Where, $k 0=2 \pi / \lambda$ is free space wave number, $\boldsymbol{n}$ is the refractive index is the phase propagation constant. The effective refractive index neff is given by

neff $=\beta / k 0$

The continuous boundary conditions of tangential components for electric and magnetic field gives

$$
\frac{-K}{a_{1}} \tan \left(k t-\tan ^{-1} \frac{a_{2}}{k}\right)-\frac{\left\{1-\left(\left(a_{1}+a_{3}\right) /\left(a_{1}-a_{3}\right)\right) e^{2 a_{1} h}\right\}}{\left\{1+\left(\left(a_{1}+a_{3}\right) /\left(a_{1}-a_{3}\right)\right) e^{2 a_{1} h}\right\}}=m \pi
$$

where $\boldsymbol{h}=$ thickness of bio layer, $\boldsymbol{m}=$ mode number is an integer After simplification, the dispersion relation in terms of ' $\boldsymbol{v}$ ' and ' $\boldsymbol{b}$ ' is obtained as

$$
\begin{aligned}
& -\sqrt{\frac{1-b}{b+a l}} \tan \left(V \sqrt{1-b}-\tan ^{-1} \sqrt{\frac{b}{1-b}}\right)-\left\{\frac{[1-(\mathrm{p})]}{[1+(\mathrm{p})]}\right\}=\mathrm{m} \pi \\
& \mathrm{P}=\left(\frac{\sqrt{b+a^{\prime}}+\sqrt{b+a}}{\sqrt{b+a^{\prime}}-\sqrt{b+a}}\right) e^{\sqrt{\frac{b+a^{\prime}}{1+a^{\prime}}}\left(2 v^{\prime}\right)}
\end{aligned}
$$

Where $\boldsymbol{v}$ is normalized frequency parameter of the three-layer slab and $v^{\prime}$ is the new normalized frequency parameter in the presence of the bio layer of thickness $\boldsymbol{h}$.

$$
\begin{gathered}
V^{\prime}=\frac{2 \pi}{\lambda} \mathrm{h} \sqrt{\left(n_{1}{ }^{2}-n^{\prime}{ }_{1}^{2}\right.} \\
a^{\prime}=\frac{\left(n_{2}{ }^{2}-n_{1}^{\prime}{ }^{2}\right)}{\left(n_{1}{ }^{2}-n_{2}{ }^{2}\right)}
\end{gathered}
$$

Four-layer structure of this can be used to model the little loss planar optical waveguides formed on silicon substrate. Among these layers, second uppermost layer with different biomaterials are coated for calculating refractive index change. The bio layer used here [4] are Si (BSA) having refractive index 3.45, silicon nitride is 2.05 and $\mathrm{Ag}$ (RI) 0.051585, Water 1.33. and $\mathrm{Al}$ is 1.4815. And this sensor is applied for refractive index measurement and detection of biomaterial applications in the body.

The Maxwell's curl equations may be expressed in Cartesian coordinates in six simple scalar equations. The remaining four are symmetric of the above four and can be obtained by exchanging the $\mathrm{x}, \mathrm{y}$, and $\mathrm{z}$ subscripts and derivatives cyclically. Maxwell's equations specify a state in which the temporal variation in the $\mathbf{E}$ field is dependent upon the spatial change of the $\mathbf{H}$ field, and vice versa.

Two among the six are:

$$
\begin{aligned}
& \frac{\partial H_{x}}{\partial t}=-\frac{1}{\mu}\left(\frac{\partial E_{y}}{\partial z}-\frac{\partial E_{z}}{\partial y}\right) \\
& \frac{\partial E_{y}}{\partial t}=-\frac{1}{\varepsilon}\left(\frac{\partial H_{x}}{\partial z}-\frac{\partial H_{z}}{\partial x}\right)
\end{aligned}
$$

The Finite difference time domain method solves the Maxwell's equations [3] first by discretizing the equations through central differences in space and time and there after solving these equations numerically in software. Using the curl of the curl identity

$\nabla \times(\nabla \times \mathbf{X})=\nabla(\nabla \cdot \mathbf{X})-\nabla^{2} \mathbf{X}$ we get

$$
\begin{aligned}
& \frac{1}{c^{2}} \frac{\partial^{2} \mathbf{E}}{\partial t^{2}}-\nabla^{2} \mathbf{E}=0 \\
& \frac{1}{c^{2}} \frac{\partial^{2} \mathbf{B}}{\partial t^{2}}-\nabla^{2} \mathbf{B}=0 \\
& c=\frac{1}{\sqrt{\mu_{0} \varepsilon_{0}}}=2.99792458 \times 10^{8} \mathrm{~m} / \mathrm{s} \\
& v_{\mathrm{p}}=\frac{1}{\sqrt{\mu_{0} \mu_{\mathrm{r}} \varepsilon_{0} \varepsilon_{\mathrm{r}}}}
\end{aligned}
$$

The best way to unravel these equations is centred on Yee's mesh and calculates $\mathbf{E}$ and $\mathbf{H}$ field components with the points on a grid which are separated by $\Delta \mathrm{x}, \Delta \mathrm{y}$, and $\Delta \mathrm{z}$ apart.

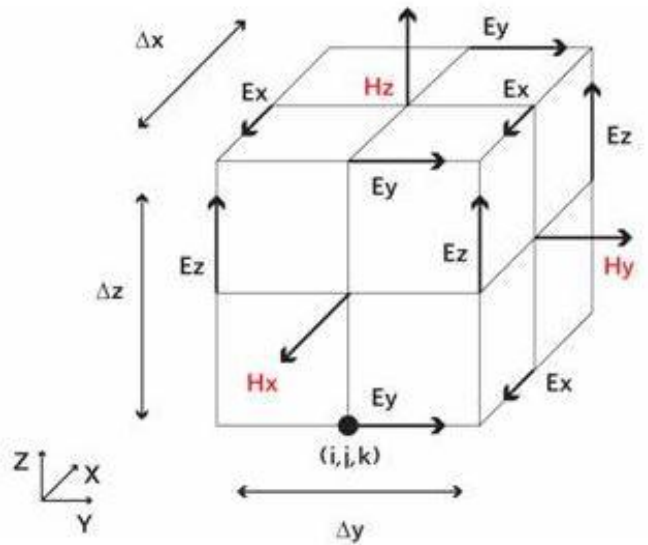

Fig 1: An Yee cell oin 3 dimensional plane corresponding to magnetic field computation.

The $\mathbf{E}$ and $\mathbf{H}$ field constituents are then interlaced in the three spatial dimensions as presented in Fig 1. Also, time is fragmented into discrete periods of $\Delta \mathrm{t}$. The $\mathbf{E}$ field constituents are then calculated at $t=n \Delta t$ and $\mathbf{H}$ fields at $t=(n+1 / 2) \Delta t$, where $n$ is an integer signifying the compute step. Maxwell's equations and wave propagation. Before we consider the surface plasmon properties let us see the Maxwell's equations which of the following form

$$
\begin{aligned}
& \nabla \cdot \mathrm{D}=\rho \\
& \nabla \cdot \mathrm{B}=0
\end{aligned}
$$

In which $\mathrm{D}, \mathrm{B}, \mathrm{E}$ and $\mathrm{H}$ represents displacement, flux density, electric field and magnetic field [5]. 
In optical field the free charge and current are assumed to be absent thereby we consider $\rho=0$ and $j=0$. In the design of optical sensors only isotropic, linear and nonmagnetic media will be taken as a consideration, thus the relation [4] among (E, D) and (H, B) can be given as

$\mathrm{D}=\epsilon 0 \epsilon \mathrm{E}$

$\mathrm{B}=\mu 0 \mu \mathrm{H}$

where $\epsilon_{0}$ is relative permittivity $=1$ and $\mu$ is the relative permeability of the medium and is equal to one for a nonmagnetic medium.These equations are solved in a leapfrog manner i.e. the $\mathbf{E}$ and $\mathbf{H}$ fields are computed alternatively at subsequent $\Delta t / 2$ intervals.

In applications involving biomolecular interactions this, SPR sensors gives high sensitivity and measurements in real time. A key portion of a FullWAVE simulation [2] is launch field, or excitation, used to excite the structure. It must be chosen in such a way that it must correspond to the required type of simulation results. A current source is designed to produce fields through the introduction of a current with in the simulation domain by introducing the current term into Maxwell's curl equations. To select this type of spatial excitation, set the Spatial Excitation Type to Current.

Since current is a vector, the direction of the current needs to be set. By default, the direction is set along the major polarization direction. To use the default direction, set the Current Direction to Auto. In 2D, the source can be considered a wire along the $\mathrm{Y}$ direction. For 2D TE simulations, the wire contains an electrical current $\left(E_{\mathrm{y}}\right)$; for $2 \mathrm{D}$ TM simulations, the wire contains a magnetic current $\left(\mathrm{H}_{\mathrm{y}}\right)$. For 3D, a user defined direction can be used. To use a custom direction, uncheck the Auto box and manually set the Direction Vector. The field profile [6] is assumed to be Gaussian in $\mathrm{X}, \mathrm{Y}$, and Z. The Gaussian width in each of these directions is defined by the values of the Width, Height, and Length fields discussed below. The other launch options are not applicable at this time. For example, a CW excitation should be preferred when steady-state performance of an assembly is required and the Pulsed excitation is preferred when the requirement is with respect to the spectral characteristics. The excitation compromises of both spatial and temporal components, and is represented as a sum of individual excitations:

$$
\phi_{L}(\mathbf{r}, t)=\sum_{i} \phi_{i}(\mathbf{r}, t)
$$

The individual excitations $\phi_{L}(\mathbf{r}, t)$ are defined as:

$$
\phi_{i}(\mathbf{r}, t)=\sqrt{P_{i}} f_{i}(\mathbf{r}) g_{i}(t) e^{i \epsilon t}
$$

Where $\theta_{i}$ and $P_{i}$ are phase and power of excitation, and $g_{i}(t)$ and $f_{i}(\mathbf{r})$ are temporal and spatial components of excitation. Each individual excitation in the above equation is defined in the same way and then combined to create the final excitation function. A continuous wave excitation type [3] is used when the requirement is the steady state performance of the design. It consists of multiplying a sinusoidal carrier by two envelope functions.

This can be expressed as

$$
g(t)=\xi_{u p}(t) \xi_{\text {davon }}(t) \sin \left[\frac{2 \pi}{\lambda}(t)+A t^{2}\right]
$$

where the wavelength $\lambda$ is set by the Wavelength field and the factor $A$, which has units of $\mu \mathrm{m}^{-2}$ produces a chirped excitation and can be set by the field Chirp Coefficient. FullWAVE monitors can perform a frequency analysis in order to obtain the spectral characteristics of a structure over a range of wavelength/frequency values. Both DFT and FFT based algorithms can be used. The names 'FFT' and 'DFT' [7] are slight misnomers as both methods are in fact discrete. However, we have used these names in order to be consistent with existing nomenclature in FDTD literature. An FFT analysis can be performed on only single-value output and is calculated at the end of a simulation.

A DFT analysis can be performed on both single-value output and spatial output at either one frequency or over a range of frequencies and is calculated as the simulation progresses. Once the Model Dimension and Polarization has been defined the default polarization is clearly defined. For example, a setting of 3D TE [2][3] corresponds to the majority of the field power in $E_{x}$ and negligible field power in $E_{y}$. The Launch Polarizer, which can be opened by clicking the Polarizer... button in the Launch Parameters dialog, allows this default behaviour to be changed to achieve a custom launch polarization.

The Launch Polarizer has two modes, each of which affects the polarization differently. The first mode is the Transformer Mode, which is the default, transforms the polarization of the launch field via a Jones Matrix T. The polarization setting of the untransformed launch field therefore affects the final polarization. It uses both built-in and custom Jones Matrices. The second mode is the Controller Mode which forces to the launch polarization to the set value while conserving the input power. The polarization setting of the untransformed launch field does not affect the final polarization. Recent study indicates that SPR sensing technique detects the alteration of RI close to surface of the sensor and it provides 2-D imaging in real-time of the biomolecular interactions on surface of the sensor. Nowadays considerable work is carried [8] in designing the SPR sensor for various applications, for this the requirement is that ultimate product must duly satisfy certain conditions like simple configuration, constant performance, robustness, great sensitivity, low cost and tiny size. Even though silver provides better sensitivity it is not preferred over the gold because it has comparatively low chemical resistance when subjected to atmosphere, therefore gold film is used as transducing layer and experiential information on biomolecular interaction on the gold surface is easily obtainable because of widespread research in current years.

FullWAVE's FFT frequency analysis [7] is expressed as a Fourier sum which is centered on a discretized Fourier Transform calculated over a finite domain:

$$
\tilde{\phi}\left(\mathbf{r}^{\prime}, \omega\right)=\alpha \sum_{t=0}^{t_{s}} \phi\left(\mathbf{r}^{\prime}, t\right) e^{i \omega t} d t
$$

where $\phi\left(\mathbf{r}^{\prime}, t\right)$ is the information given by the monitor, $t_{s}$ is the stop time of simulation, and $w$ is the frequency. The normalization factor $\alpha$ is chosen such that the length of the time window will not change the value of the integral and depends on the Excitation type chosen. The complete time response of any selected singlevalue output is recorded and the Fourier sum is computed when the simulation has completed. This type of frequency output is optimized for simulation speed.

FullWAVE [7] computes the EM field as a function of space and time for a random refractive index structure for the reaction to an excitation and displays this field at the time intervals. To record this field information as a function of time or frequency for post processing and analysis purposes, we introduce the concept of monitors. A monitor archives both the field values and common EM quantities such as energy or power density as a function of frequency or time. This yield can be further used for postprocessing if needed. 
FDTD is used to compute the band gap in the photonic crystal. This method [9] is a straight forward result to Maxwell's time dependent curl equations. The FDTD method finds the time evolution of EM wave by discretizing the Maxwell's equations. FDTD utilizes 2nd order and central-difference estimates for space and time derivatives straightly to corresponding differential operators of curl equations. In FDTD method [5] theoretically there is no restriction in solving the Maxwell's equations. The main reason why FDTD method is preferred over the other approximation techniques is other methods fails abide to structure and geometry whereas FDTD doesn't. In order to implement the FDTD method MEEP tool is used.

\section{Sensor Design}

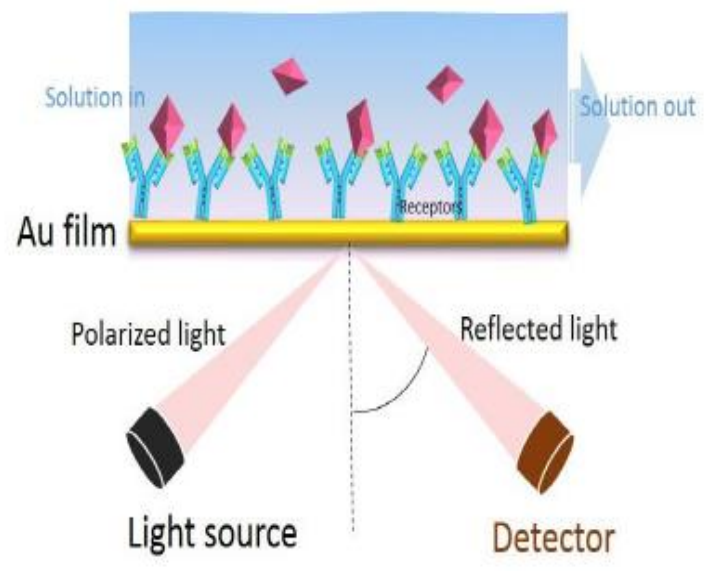

Fig 2: Sensor Structure

When monochromatic (single wavelength), polarized light [10] strikes the interface of two media, the light is partially reflected. In SPR, the prism is covered with a gold film. The free conduction electrons in gold produces periodic oscillations which is called plasma waves. The particle name for plasma is called Plasmon. The proposed design given in the figure 2 is an SOI based surface plasmonic resonance biosensor which is based on interferometer where metal dielectric interface at the input port act as a source for Plasmon poloritons whose impulsive responses are subjected to variation in the effective refractive index(RI). Here the selected metal is Guld embedded in Titanium oxide and $\mathrm{SiO} 2$ as dielectric material sandwiched between the $\mathrm{ZnS}-\mathrm{SiO} 2$ and metal (Ti).

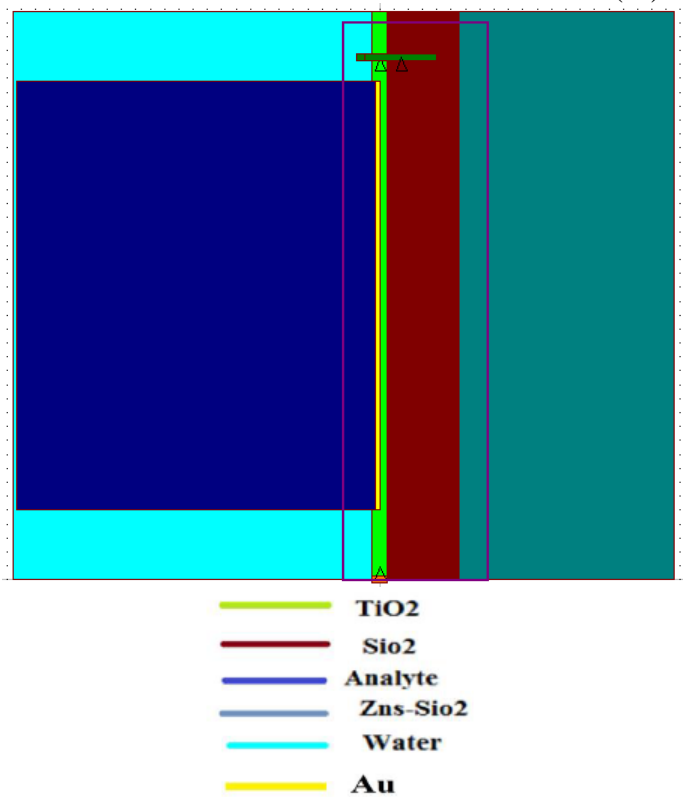

Fig 3: Design structure in Rsoft
RSOFT photonic suit [3] products are used to design and analyse the optical components, telecommunication systems and nanoscale optical structures. Within the physical layer the simulation tool suit allows the user to develop the design and simulate both the passive and active photonic optic electronic components.

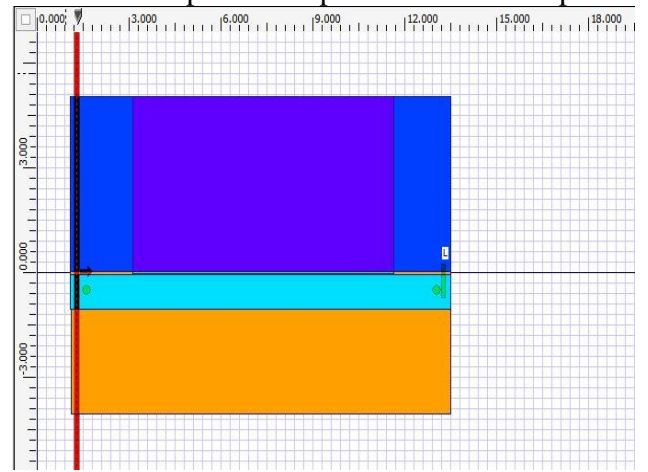

Fig 4: Design structure in Optifdtd

The structure design includes segments of waveguides. These waveguides include metals that has the capacity to absorb the light that is the source. The incident light is absorbed by the electrons in the metal to constitute the storage. There segments are laid parallel to each other. A monitor that is a spectrum analyser is placed at the end of the waveguide to capture the reflected waveforms. The RSOFT has different simulation engine tool used for different models of optics. The OPTIWAVE OPTIFDTD software is also been used to obtain the $3 \mathrm{~d}$ modeling of the design. In this software the refractive index of the design is been used for the simulation.

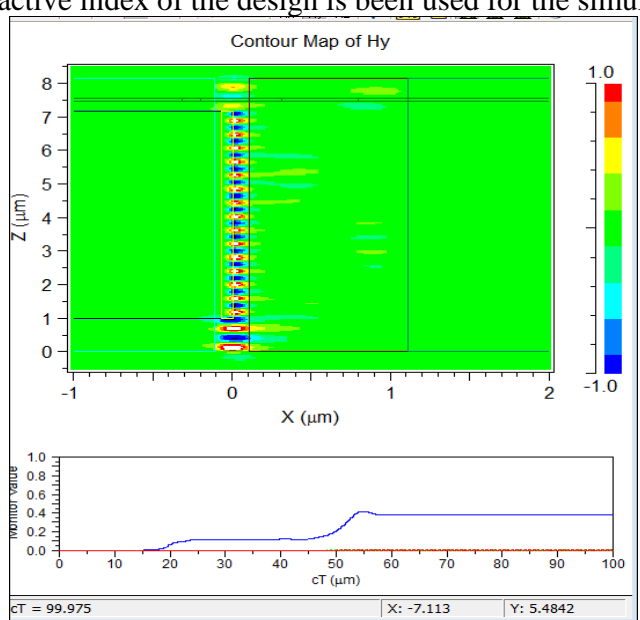

Fig 5: Output in Rsoft

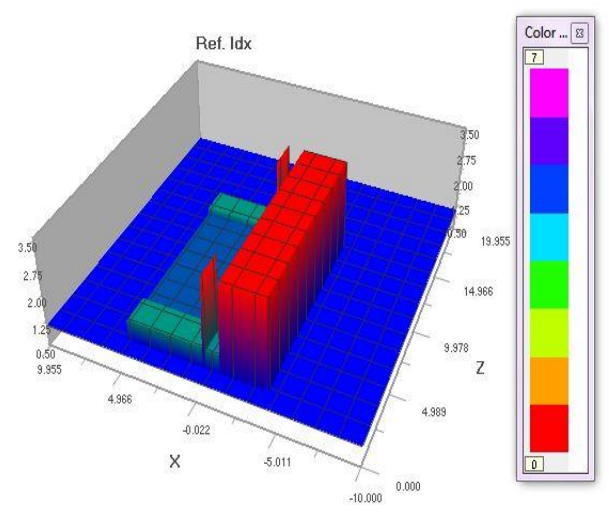

Fig 6: 2D Refractive Index Output

The design description for the sensor design is given in the figure 7. 


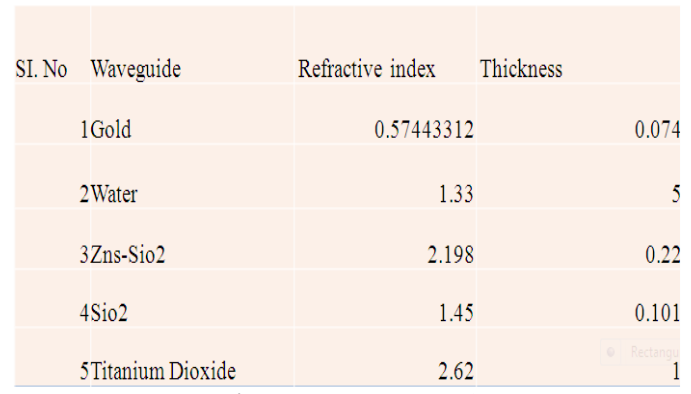

Fig 7: Parameters used

For the SPR structure the same design is been done using the Optifdtd software. The Optiwave software is a finite difference time domain tool, it helps in plotting of the pointing vector and the frequency spectrum analysis are plotted. The designing of the SPR using Optifdtd tool has a profiles and materials section where the output is being plotted and the analysis is being calculated and the designing of the slab layers is computed. The gold is being used as the sensing layer of the design. The bacillus cereus is the solution which will be placed on top of the optical sensor top layer. The sensing layer reacts with the solution to form the plasmons. This plasmons are observed by the observation point. The observation point has the data components such as $\mathrm{Hy}, \mathrm{Hx}$ and $\mathrm{Hz}$ magnetic components and Ey, Ex and Ez electrical parameters. The both electrical and magnetic properties will be used in the plotting of the graph. The Optifdtd and the Rsoft both tool is used to simulate the design. The contour map of figure 3 depicts the transmission of light at the metal dielectric interface. The monitor window below the contour graph portrays the instantaneous amplitude value with respect to time.

\section{Simulation Results}

RSOFT and OPTIFDTD is used for developing large photonic devices, it works on FDTD techniques for the simulation principle. Designs such as SPR, band gaps can be developed using full wave tool. The output simulation spectrum is obtained using RSOFT.

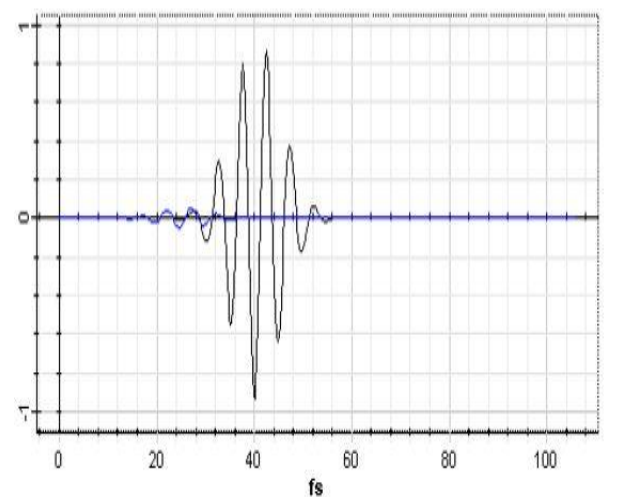

Fig 8: Time domain response in observation point in Optifdtd

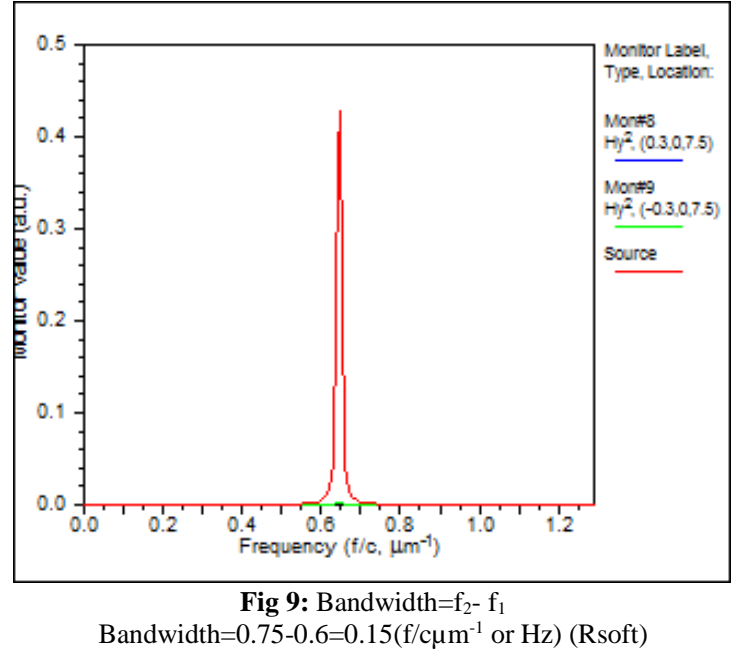

As refractive index of bio-sample taken into consideration will change according to different concentrations in water this shifts transmission frequencies and transmitted output power levels, which may be observed from transmission spectrums which act as monogram for designed sensor structure.

The figure 8 and 9 explains about the time domain and operating frequency range of the sensor design which is from 0.6 tom 0.8 $\mathrm{HZ}$.

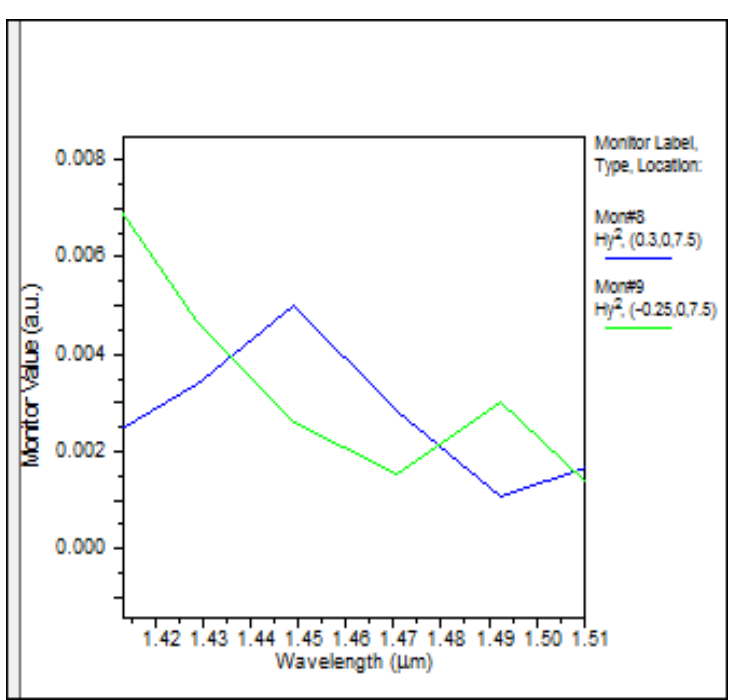

Fig 10: Wavelength response

The figure 10 gives the wavelength response with respect to amplitude for two monitors, where one monitor kept above the metal dielectric interface and one kept below the metal dielectric interface the responses from the monitors when compared shows shift in the wavelength of $0.02 \mu \mathrm{m}$.

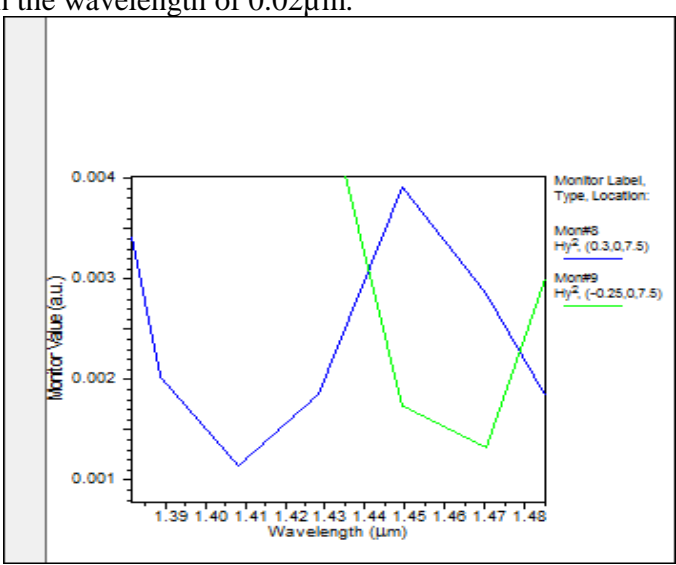

Fig 11: Transmission spectrum of the sample in Rsoft 
The figure 11 demonstrates the design simulated with samples. The MOST Analysis is being performed for the two different samples. By this analysis the sensitivity of the graph is been found. So, it can be concluded that sensitivity of sensor is found to be $105.565 \mathrm{~nm} / \mathrm{RIU}$.

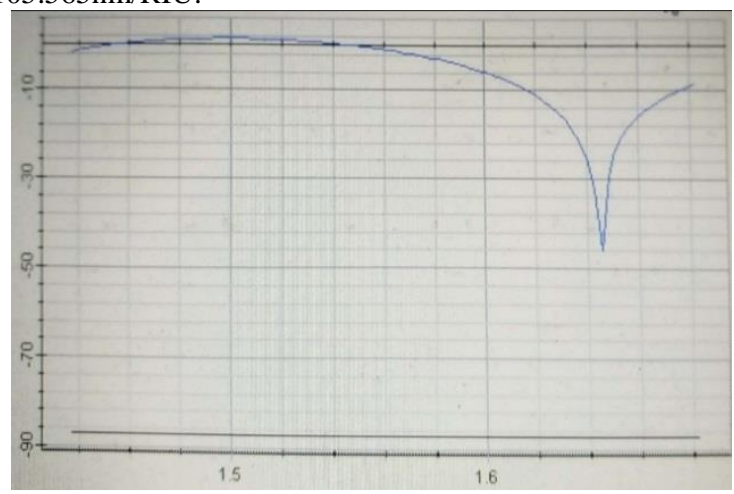

Fig 12: Transmission spectrum of the sample in OPTIFDTD

The figure 12 illustrates the transmission spectrum of the sample in the Optifdtd. The idea of simulation concept is to use the finite difference time domain method to obtain the near field pattern and the near field transmission and reflection. The enriched transmission is intermediated by the surface plasmon polaritons (SPPs) that unveil resonances arranged by the periodicity. The transmission function is acquired from the Observation Point analysis. The point detector is put into the waveguide centre where the value is obtained.

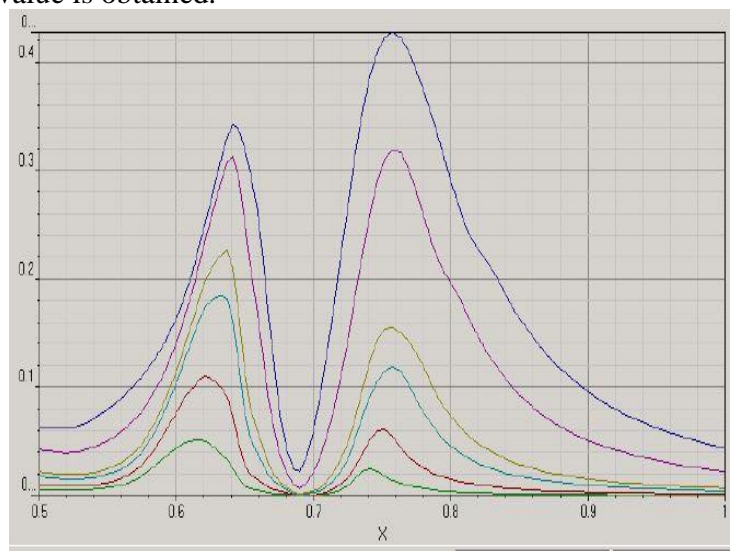

Fig 13: Power transmission spectrum in OPTIFDTD

Figure 13 illustrates the Power transmission spectrum of the design in Optifdtd. Observation Area from the Optifdtd toolbar is used to compute power transmission ratio. It is the the distribution of energy of the waveforms among its different frequency components. The respective simulation is being plotted for the different sample wavelengths. The power transmission spectrum is be obtained after simulation.

\section{Conclusion}

The Surface Plasmon resonance is the most widely adopted technique to analyze the biomolecular interactions and also detecting the components of interest present in the samples over the metal surface in real time SPR is a real-time, free labelling technique which is made possible through highly available optical sensors. Rsoft Tool and OPTIFDTD Tool is used to design the Surface Plasmon Resonance. The current work has been done on finding the micro bacillus cereus in the blood whose presence can eventually damage the wall of the red cells in the human blood as B.Cereus are one of the kind of hemolytic bacteria. The sensitivity of the designed sensor has been calculated and found to be $105.565 \mathrm{~nm} / \mathrm{RIU}$. The analysis above is carried out by varying refractive index which is resulted in the proper functioning of the device by producing the shift in the wavelength thus making the proposed design compact and relatively easy to fabricate using optical lithography as compared to the conventional SPR sensor. Also, metal which has a complex refractive index and as a result though SPR waveguide produces large confinement it suffers from propagation loss. Thus, there is need to develop different plasmonic waveguide and has to identify the best waveguide design that can be used for the various application. The sensor was designed on the basis of an SOI structure in order to obtain high confinement of light compared to another sensor. The simulation was done using an optical simulator tool with an input source wavelength of $1.550 \mathrm{um}$. The signature graphs for all the components were obtained, the resonant frequency.

\section{References}

[1] N Gurler, L Oksuz, M Muftuoglu, Fd Sargin2 and SK Besisik," Bacillus Cereus Catheter Related Bloodstream Infection in a Patien with acute lymphoblastic leukemia, mediterranean journal of hematology and infectious diseases, issn 2035-3006.

[2] Edward J. Bottone" Bacillus cereus, a Volatile Human Pathogen' CLINICAL MICROBIOLOGY REVIEWS, Apr. 2010, p. 382-398

[3] Mahoko Ikeda, Yuka Yagihara, Keita Tatsuno, Mitsuhiro Okazaki, Shu Okugawal and Kyoji Moriya," Clinical characteristics and antimicrobial susceptibility of Bacillus cereus blood stream infections" Ikeda et al. Ann Clin Microbiol Antimicrob (2015) $14: 43$

[4] Homola J, `Ctyrock'y J, Skalsk'y M, Hradilov'a J and Kol'a rov'a $\mathrm{P}$, "A Surface Plasmon Resonance Based IntegratedOptical Sensor," Sens. Actuators B,38-39, 286 - 290 (1997)

[5] Dost'alek J, `Ctyrock'y J, Homola J, Brynda E, Skalsk'y M, Nekvindov'a P, 'Spirikov'a J,

[6] "Skvor J and Schr"ofel J,"Surface Plasmon Resonance Biosensor based on Integrated Optical Waveguide," Sens. Actuators B, 76, 8 12(2001)

[7] Kolstø AB, Tourasse NJ, Økstad OA. What sets Bacillus anthracis apart from other Bacillus species? Annu Rev Microbiol. 2009;63:451-76.

[8] De Ronde, F. R. and S. Shammas, IMIC band ${ }^{-}$lters using open-ring resonators," Proc. $4^{\text {th }}$ Eur. Microwave Conf., 531 $\{535$, Montreux, Switzerland, Sept. 1974. 2. Lo, Y. T., D. Solomon, and W. F. Richards, ITheory and experiment on microstrip antennas," IEEE Transaction on Antennas and Propagation, Vol. 27, 137\{145, 1979.

[9] Wol®, I. and V. K. Tripathi, IThe microstrip open-ring resonator," IEEE Transactions on Microwave Theory and Techniques, Vol. 32, No. 1, Jan. 1984

[10] Elhawil, A., J. Stiens, C. De Tandt, W. Ranson, and R. Vounckx, $\backslash$ An equivalent circuit

model of single circular open-ring resonators," IEEE Journal of Selected Topics in Quantum

Electronics, Vol. 16, No. 2, Mar./Apr. 2010. 